\title{
Auditory Hallucinations Elicit Similar Brain Activation in Psychotic and Nonpsychotic Individuals
}

\author{
Kelly M. J. Diederen ${ }^{*}$, Kirstin Daalman ${ }^{1}$, Antoin D. de Weijer ${ }^{1}$, Sebastiaan F. W. Neggers ${ }^{1}$, Willemijn van Gastel ${ }^{1}$, \\ Jan Dirk Blom ${ }^{2,3}$, René S. Kahn1, and Iris E. C. Sommer ${ }^{1}$ \\ ${ }^{1}$ Department of Psychiatry, Neuroscience Division, Rudolf Magnus Institute for Neuroscience, University Medical Center Utrecht, B01.206, \\ Heidelberglaan 100, 3584 CX, The Netherlands; ${ }^{2}$ Parnassia Bavo Group, The Hague, The Netherlands; ${ }^{3}$ Department of Psychiatry, \\ University of Groningen, Groningen, The Netherlands \\ *To whom correspondence should be addressed; tel: +31887556370, fax: +31887555509, e-mail: K.M.J.Diederen@umcutrecht.nl
}

While auditory verbal hallucinations (AVH) are most characteristic for schizophrenia, they also occur in nonpsychotic individuals in the absence of a psychiatric or neurological disorder and in the absence of substance abuse. At present, it is unclear if AVH in these nonpsychotic individuals constitute the same phenomenon as AVH in psychotic patients. Comparing brain activation during AVH between nonpsychotic and psychotic individuals could provide important clues regarding this question. 21 nonpsychotic subjects with AVH and 21 matched psychotic patients indicated the presence of AVH during 3T functional magnetic resonance imaging (fMRI) scanning. To identify common areas of activation during the experience of $\mathrm{AVH}$ in both groups, a conjunction analysis was performed. In addition, a 2 -sample $t$-test was employed to discover possible differences in AVH-related activation between the groups. Several common areas of activation were observed for the psychotic and nonpsychotic subjects during the experience of AVH, consisting of the bilateral inferior frontal gyri, insula, superior temporal gyri, supramarginal gyri and postcentral gyri, left precentral gyrus, inferior parietal lobule, superior temporal pole, and right cerebellum. No significant differences in AVH-related brain activation were present between the groups. The presence of multiple common areas of AVH-related activation in psychotic and nonpsychotic individuals, in the absence of significant differences, implicates the involvement of the same cortical network in the experience of $\mathrm{AVH}$ in both groups.

Key words: auditory verbal hallucinations/psychosis/ schizophrenia/functional magnetic resonance imaging/ continuum hypothesis of psychosis

\section{Introduction}

Auditory verbal hallucinations (AVH) are common in schizophrenia patients but also occur in other psychiatric disorders including schizotypal and borderline personality disorder, bipolar disorder, and psychotic depression. ${ }^{1,2}$ Moreover, AVH are observed in nonpsychotic individuals, in the absence of delusions, affective, and negative symptoms. ${ }^{3-5}$ These observations led to the formulation of the continuum hypothesis of psychosis, which states that symptoms observed in patients with a psychotic disorder are also apparent in nonclinical populations. ${ }^{6,7}$ However, it remains unclear if AVH in nonpsychotic individuals are the same symptom as AVH in psychotic patients. Similarities in a number of phenomenological characteristics of AVH in psychotic and nonpsychotic individuals suggest that AVH are indeed the same phenomenon in both groups. ${ }^{8,9}$ For instance, experienced location, loudness, reality, number of voices, and personification of the AVH were shown to be similar for both groups. ${ }^{8,9}$ However, differences in several other AVH characteristics may shed doubt on classifying AVH in nonpsychotic individuals as "actual" hallucinations; frequency, age of onset, emotional valence of the content, associated distress, and the degree of control individuals experience over their AVH were found to differ between nonpsychotic and psychotic subjects. $^{8-10}$ As both differences and similarities in phenomenological characteristics of AVH were observed in nonpsychotic and psychotic individuals, ${ }^{8,9}$ it remains unclear if $\mathrm{AVH}$ in nonpsychotic individuals result from the same mechanism as AVH in psychotic patients. If these hallucinations are caused by comparable mechanisms in both groups, one should observe the same pattern of brain activation during AVH. Several studies investigated AVH-related brain activation in psychotic patients, primarily reporting activation of bilateral frontal and temporoparietal areas. ${ }^{11-14}$ Thus far, only one study visualized AVH-related brain activation in nonpsychotic subjects, revealing activation in bilateral frontal and temporoparietal areas as well as motor

(C) The Author 2012. Published by Oxford University Press on behalf of the Maryland Psychiatric Research Center. All rights reserved.

For permissions, please email: journals.permissions@oup.com 
areas. ${ }^{15}$ However, this study included only 7 subjects and did not compare AVH-related brain activation in their group of nonpsychotic subjects to that of psychotic patients. The aim of the present study was to identify commonalities and/or differences in brain activation during AVH between psychotic and nonpsychotic individuals with $\mathrm{AVH}$ in samples that are large enough to enable group-wise analysis.

\section{Methods}

\section{Participants}

Twenty-one nonpsychotic individuals with AVH and 21 psychotic patients participated in this study. Nonpsychotic subjects with AVH were recruited via a website: www.verkenuwgeest.nl (explore your mind). An extended description of the recruitment and selection procedure is provided in prior studies by our group..$^{4,8,16,17} \mathrm{In}$ short, visitors of this website filled out a questionnaire based on the Launay Slade Hallucination Scale $\left(\right.$ LSHS $^{18}$ ), which is a self-report questionnaire designed to quantify the tendency to hallucinate in healthy individuals. Subjects with high scores on item 8 and 12 of the LSHS, tapping into auditory hallucinations, were invited to the University Medical Center Utrecht to undergo detailed psychiatric assessment.

Psychotic patients were selected from a larger group ${ }^{19}$ to match the healthy individuals for demographic factors, such as age, sex, and handedness but also for the total duration of the AVH, the mean duration of the AVH, and the number of $\mathrm{AVH}$ experienced during the functional magnetic resonance imaging (fMRI) scans. Patients were recruited from the Department of Psychiatry, University Medical Center Utrecht and the Parnassia Bavo Group in The Hague, The Netherlands. All psychotic patients were diagnosed by an independent psychiatrist using the Comprehensive Assessment of Symptoms and History interview $\left(\mathrm{CASH}^{20}\right)$. Ten patients were diagnosed with schizophrenia, 2 with schizoaffective disorder, and 9 with psychosis Not Otherwise Specified (NOS). The main difference between patients diagnosed with schizophrenia and schizoaffective disorder and patients diagnosed with psychosis NOS was that the first groups scored higher on items addressing negative symptoms and general psychopathology. All patients, including patients diagnosed with psychosis NOS, presented with additional positive symptoms including delusions and disorganization.

General inclusion criteria were (1) voices should be distinct from thoughts and have a perceptual quality, (2) voices should be experienced at least once a month, (3) no chronic somatic disorder, and (4) no drug use for at least 1 month prior to the assessment. To confirm the absence of drug use, urine samples were collected and tested for opiates, amphetamines/XTC, cocaine, and cannabis. Furthermore, a number of additional scanrelated inclusion criteria were used. These additional criteria were (5) voices should be present with a frequency of at least $4 \mathrm{AVH}$ episodes per scan (8 minutes), (6) and with a minimum total duration of fifty seconds. Finally, (7) hallucinations should be indicated correctly, ie, each onset (balloon-squeeze) should be followed by a clear offset (balloon-release).

Additional criteria for the nonpsychotic subjects consisted of (1) no psychiatric disorder other than anxiety or depressive disorder in full remission according to Diagnostic and Statistical Manual of Mental Disorders, Fourth Edition, (DSM-IV) diagnostic criteria assessed by an independent psychiatrist using the $\mathrm{CASH}^{20}$ and Structured Clinical Interview for personality Disorder (SCID-II ${ }^{21}$ ) interview and (2) no drug or alcohol abuse prior to the first AVH experience.

In total, 42 nonpsychotic individuals with AVH participated in the hallucination scans. Twenty-one of these individuals were excluded from analyses because they did not meet inclusion criteria 5 or 6 . Of these 21 , 7 subjects experienced no AVH at all during the hallucination scan.

Nonpsychotic subjects with AVH were not diagnosed as Psychosis NOS because they were not bothered by these AVH and showed no social or professional dysfunction. Although the nonpsychotic individuals with hallucinations did not have any clinical delusions, they did score significantly higher on the Schizotypal Personality Questionnaire $\left(\mathrm{SPQ}^{22}\right)$ than a group of matched healthy control subjects. ${ }^{4}$ In addition, the combination of hallucinations (perceptual aberrations) and magical ideation present in most nonpsychotic individuals with AVH made them score on at least 3 items on the DSM-IV criteria for schizotypal personality disorder. However, social capacity and affect were found to be adequate as determined by a trained psychiatrist using the CASH and the global assessment of functioning $\left(\mathrm{GAF}^{23}\right)$ scale.

Group-wise matching was used to match the group of psychotic patients to the nonpsychotic individuals with AVH. After selecting the 21 nonpsychotic individuals who met all inclusion criteria, we inspected our database, which contains hallucination scans of psychotic patients, in order to select a group of patients comparable to the nonpsychotic individuals. Psychotic patients included in this database are described in detail in a previous study by our group. ${ }^{19}$ For the matching, the following order was used: sex, handedness, age, years of education, total duration of the hallucinations during the scans, number of durations during the scans, and average duration of a hallucination during the scans. If the groups differed significantly on one of these variables, we tried minimizing these differences, being less strict with respect to one of the other variables. Unfortunately, we could not adequately match the groups with respect to years 
Table 1. Medication Use and Scores on the PANSS for the Psychotic Patients

\begin{tabular}{llll}
\hline & $N$ & Mean (SD) & $\begin{array}{c}\text { Median } \\
\text { (Range) }\end{array}$ \\
\hline $\begin{array}{l}\text { Antipsychotic medication } \\
\quad \text { First generation }\end{array}$ & 9 & & \\
$\quad$ Second generation & 9 & & \\
$\quad$ No antipsychotic & 3 & & \\
PANSS scores & & & \\
$\quad$ Total PANSS & 19 & $65.32(15.67)$ & $67(53)$ \\
$\quad$ Positive PANSS & 19 & $16.53(4.03)$ & $17(14)$ \\
$\quad$ Negative PANSS & 19 & $15.84(4.99)$ & $15(14)$ \\
$\quad$ General psychopathology & 19 & $32.95(8.42)$ & $34(25)$ \\
\hline
\end{tabular}

Note: Abbreviations: $N$, number; PANSS, Positive and Negative Syndrome Scale.

of education as most patients dropped out of school as a result of the onset of psychiatric symptoms.

The groups did not differ significantly with respect to age, sex, and handedness; however, years of education were significantly lower for the psychotic patients. In table 1, medication use and scores on the Positive and Negative Syndrome Scale ${ }^{24}$ are listed for the psychotic patients.

Table 2 provides a demographic description of all participants including the characteristics of the voices in the last 3 months according to the Psychotic Symptom Rating Scales (PSYRATS), ${ }^{25}$ scores on the GAF, and scores on the SPQ for the group of nonpsychotic individuals with AVH. This study was approved by the Human Ethics Committee of the University Medical Center Utrecht. After complete description of the study to the subjects, written informed consent was obtained.

\section{Experimental Design and Data Acquisition}

During fMRI acquisition, participants indicated the presence of AVH by balloon-squeezes. Activation maps were obtained using a Philips Achieva 3 Tesla Clinical MRI scanner. Eight hundred blood-oxygenation-level-dependent (BOLD) fMRI images were acquired per patient with the following parameter settings: 40 (coronal) slices, TR/TE $21.75 / 32.4 \mathrm{~ms}$, flip angle $10^{\circ}$, field of view (FOV) $224 \times 256 \times 160$, matrix $64 \times 64 \times 40$, voxelsize $4 \mathrm{~mm}$ isotropic. This scan sequence achieves full brain coverage within $609 \mathrm{~ms}$ by combining a 3D-PRESTO pulse sequence with parallel imaging (SENSE) in 2 directions using a commercial 8-channel SENSE headcoil. ${ }^{26}$ Since these PRESTO SENSE images have little anatomical contrast, 40 identical scans, but with a flip angle of $27^{\circ}$ (fa27), were acquired to improve realignment and coregistration during the preprocessing. After the functional scans, a high-resolution anatomical scan, with the following parameters: TR/TE: $9.86 / 4.6 \mathrm{~ms}, .875 \times .875 \times 1$ vox- els, flip angle $8^{\circ}$, FOV $224 \times 160 \times 168.00,160$ slices was acquired to improve localization of the functional data.

\section{Data Analysis}

Preprocessing and data analysis was conducted with statistical parametric mapping (SPM5; Welcome Department of Cognitive Neurology). Preprocessing included within-subject image realignment with the mean fa27 as the reference to correct for the effects of head motion, coregistration of the mean fa27 and the T1 weighted anatomical image, and spatial normalization to a standard Montreal Neurological Institute template. Finally, images were smoothed using an $8-\mathrm{mm}$ full width at half maximum Gaussian kernel. To compare activation during hallucination periods with nonhallucination periods, a model was created using balloon-squeezes as hallucination onsets and time between squeezes and releases as the durations. Functional images were analyzed on a voxel by voxel basis using multiple regression analysis ${ }^{27}$ with one factor coding for activation (hallucination vs nonhallucination). This model was convolved with the standardized hemodynamic response function from SPM5 to introduce typical delays of fMRI responses and fitted to the data using general linear model estimation. To model movement artifacts the realignment parameters were entered as covariates.

To identify common areas of activation for the experience of $\mathrm{AVH}$ in the 2 groups, a conjunction analysis (conjunction null ${ }^{28}$ ) was performed. For this analysis, the following steps were taken. First, one sample $t$-tests, comparing brain activation during hallucinations (balloon-squeeze) to brain activation during baseline (no balloon-squeeze), were performed on individual fMRIBOLD signal change maps to enable group-wise analyses for the psychotic and nonpsychotic subjects, separately. Subsequently, to determine which voxels were significantly activated in both groups, 1 -sample $t$-tests of the psychotic and nonpsychotic individuals were overlaid on one another.

Furthermore, to identify possible differences in brain activation during AVH between the 2 groups, a 2-sample $t$-test was employed. It was hypothesized that possible differences as well as similarities in brain activation would be present in regions previously reported to be involved in the experience of AVH. To enable hypothesis-driven analyses for the conjunction analysis as well as for the 2-sample $t$-test, a small volume correction for multiple comparisons was applied. The small volume contained regions previously reported to be involved in the experience of AVH. ${ }^{11-14}$ These regions, comprising the bilateral inferior frontal gyri, insula, superior and middle temporal gyri (including the superior and middle temporal pole), supramarginal gyri, precentral and postcentral gyri, cerebellum, hippocampus, and parahippocampal gyrus were defined using the Automated 


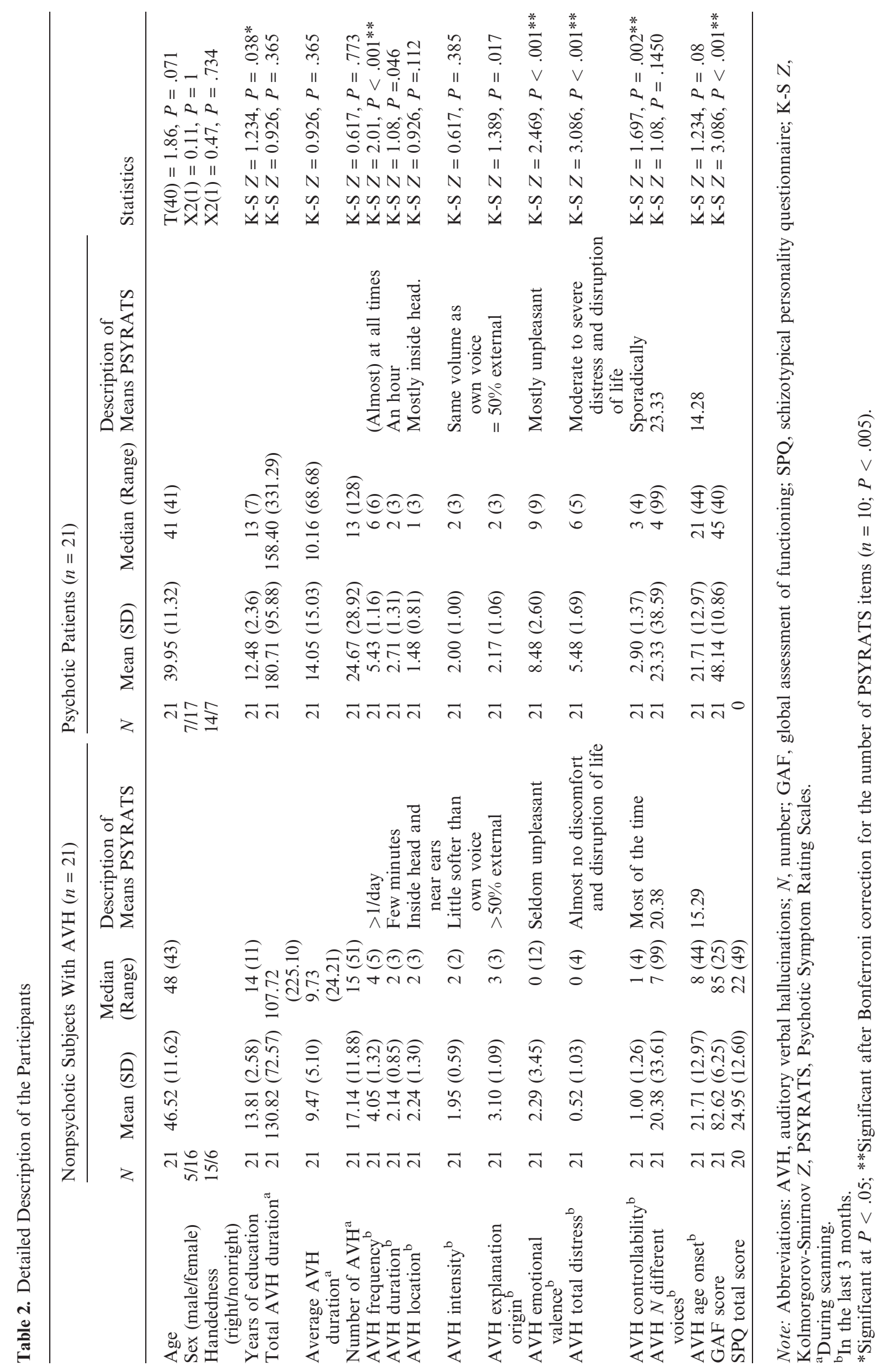




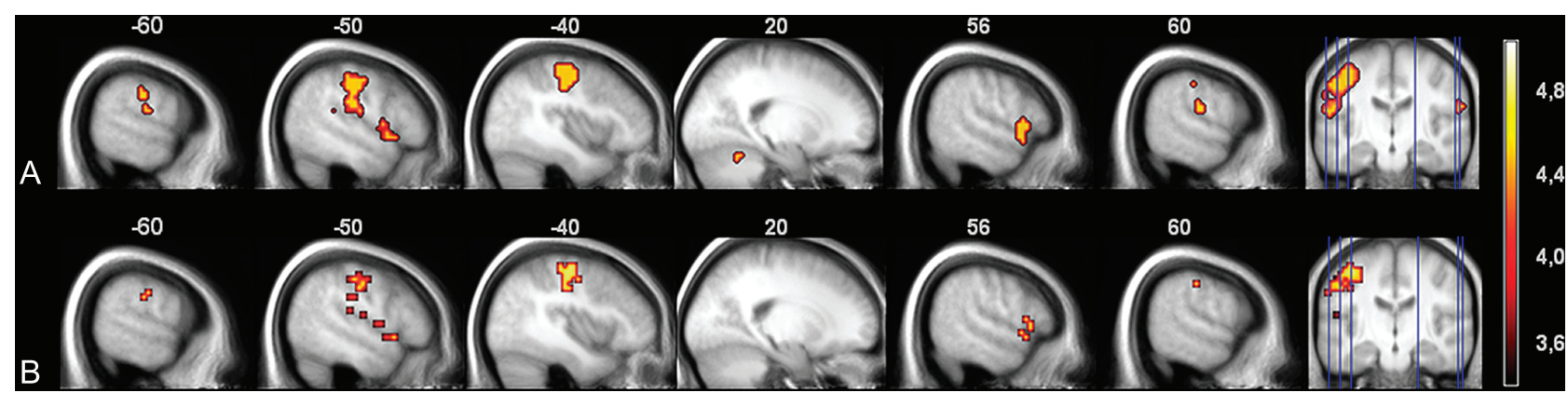

Fig. 1. SPM(T)'s for the conjunction analysis revealing brain regions significantly activated during the experience of AVH in both psychotic and nonpsychotic individuals with AVH. (A) Areas significantly activated within a priori hypothesized regions. (B) Areas significantly activated within all gray matter voxels in the brain Thresholded at $P=.05$ False Discovery Rate (FDR) corrected for multiple comparisons. Abbreviations: SPM(T)'s, spatial parametric mapping T-values; AVH, auditory verbal hallucinations.

Anatomical Labeling atlas. ${ }^{29}$ Moreover, to identify potential similarities and differences in brain activation outside the selected regions, an additional exploratory conjunction analysis and independent samples $t$-test were performed, testing all gray matter voxels within the brain. All analyses were thresholded at $P=.05$, False Discovery Rate $^{30}$ corrected for all voxels within the regions of interest for the hypothesis-bases analysis and for all voxels within gray matter for the exploratory analysis. In addition, an extended threshold of 5 voxels was used.

In addition, to determine if possible differences in lateralization of language areas implicated in $\mathrm{AVH}$ would be present between the psychotic and nonpsychotic individuals with $\mathrm{AVH}$, lateralization indices were calculated on individual $t$-tests and compared between the groups. For this purpose, a mask was created using the Anatomical Automatic Labeling ${ }^{29}$ atlas comprising the main areas where language processing is thought to be mediated and their contralateral homologues. ${ }^{14,16}$ Language areas consisted of the inferior frontal triangle, the insula, the middle temporal gyrus, the superior temporal gyrus, the supramarginal gyrus, and the angular gyrus. Lateralization indices were defined as the difference in "thresholded" signal intensity changes in the left vs the right hemisphere (within the selected language regions) divided by the total sum of thresholded signal intensity changes. Using this method, activity measures are based on signal intensity changes in those voxels that exceed a predefined activation level, as recommended by Jansen and colleagues. ${ }^{31}$ Differences in lateralization indices between the 2 groups were compared by means of Kolmogorov-Smirnov test. Finally, to replicate the finding of a significant negative correlation between emotional valence of the AVHcontent and lateralization indices within language areas by Sommer and colleagues, ${ }^{14}$ lateralization indices were correlated with the emotional valence of the content measured with the PSYRATS and tested for significance using spearman's rho.

\section{Results}

The hypothesis-based conjunction analysis revealed several areas that were significantly activated during the experience of $\mathrm{AVH}$ in both groups including the bilateral inferior frontal gyri, insula, superior temporal gyri, supramarginal gyri and postcentral gyri, left precentral gyrus, inferior parietal lobule, superior temporal pole, and right cerebellum. Except for the right cerebellum, in which no significant activation was observed, the exploratory analysis yielded activation of the same regions, albeit cluster sizes of significant voxels were smaller. Figure 1A shows the SPM(T)'s of all significant local maxima for the conjunction analysis in a priori hypothesized regions and figure 1B shows significant activation within all gray matter voxels in the brain. Table 3 shows the coordinates, $t$-values and cluster size of all significant local maxima for the conjunction analysis in a priori hypothesized regions. The 2-sample $t$-test revealed no significant difference in activation during AVH between the groups neither in a priori hypothesized regions nor in all gray matter voxels in the brain.

In addition, no significant difference $(P=0.6)$ in lateralization indices could be observed between the nonpsychotic individuals (mean $=-0.09 ; \mathrm{SD}=0.29$; range $=1.36$ ) and the psychotic patients (mean = $-0.04 ; \mathrm{SD}=0.18$; range $=0.67$ ). Finally, lateralization indices of hallucinatory activation were not significantly correlated to the emotional valence of the content in either the nonpsychotic (spearman's rho $=0.17, P=$ .46) or in the psychotic individuals (spearman's rho $=$ $0.13, P=.58)$.

\section{Discussion}

This study investigated brain activation during $\mathrm{AVH}$ in 21 nonpsychotic and 21 psychotic individuals. While several common areas of activation were present for the psychotic and nonpsychotic subjects, no significant differences in brain activation could be observed between 
Table 3. $T$-Values, Cluster Sizes, and Locations of Local Maxima for the Conjunction Analysis in a priori hypothesized regions

\begin{tabular}{|c|c|c|c|c|}
\hline $\begin{array}{l}\text { Cluster } \\
\text { size }\end{array}$ & $\begin{array}{l}P \\
\text { Value }\end{array}$ & $\begin{array}{l}T \\
\text { Value }\end{array}$ & $\begin{array}{l}\text { MNI } \\
\text { Coordinates }\end{array}$ & Brain Regions \\
\hline \multirow[t]{3}{*}{152} & .008 & 5.19 & $-44,-20,44$ & $\begin{array}{l}\text { L postcentral gyrus/ } \\
\text { supramarginal gyrus }\end{array}$ \\
\hline & .017 & 4.18 & $-52,-20,24$ & $\begin{array}{l}\text { L precentral gyrus/ } \\
\text { superior temporal } \\
\text { gyrus }\end{array}$ \\
\hline & .023 & 3.82 & $-60,-24,32$ & $\begin{array}{l}\mathrm{L} \text { inferior parietal } \\
\text { lobule }\end{array}$ \\
\hline 13 & .022 & 3.93 & $56,12,-4$ & $\begin{array}{l}\text { R superior temporal } \\
\text { gyrus/inferior frontal } \\
\text { gyrus/insula }\end{array}$ \\
\hline 20 & .022 & 3.90 & $-52,8,4$ & $\begin{array}{l}\text { L inferior frontal gyrus/ } \\
\text { insula/superior temporal } \\
\text { gyrus/superior temporal } \\
\text { pole }\end{array}$ \\
\hline 7 & .025 & 3.65 & $60,-16,20$ & $\begin{array}{l}\text { R postcentral gyrus/ } \\
\text { supramarginal gyrus }\end{array}$ \\
\hline 5 & .028 & 3.46 & $20,-56,-20$ & $\mathrm{R}$ cerebellum \\
\hline
\end{tabular}

Note: Thresholded at $P=.05$, FDR corrected with an extended threshold of 5 voxels. Abbreviations: AVH, auditory verbal hallucinations; MNI, Montreal Neurological Institute; L, left; $\mathrm{R}$, right; FDR, false discovery rate.

the groups. In addition, no significant differences in lateralization of language activity could be observed between the psychotic and nonpsychotic individuals. Finally, no significant correlation was present between lateralization indices and the emotional valence of the AVH-content, in either group.

Psychotic as well as nonpsychotic subjects with AVH activated the bilateral inferior frontal gyri, insula, superior temporal gyri, supramarginal gyri, postcentral gyri, left precentral gyrus, inferior parietal lobule, superior temporal pole, and right cerebellum. These areas were discovered with a conjunction analysis, which identifies common areas of activation by finding areas that are significantly activated in each group. ${ }^{28}$ The result of several common area of activation in the psychotic and nonpsychotic individuals implicates involvement of the same network in the experience of AVH in both groups. However, to what extent a similar network is involved, is unclear as, during $\mathrm{AVH}$, no significant differences could be observed between the groups. Future studies may aid in identifying possible differences in AVH-related brain activation between these individuals. In addition, while this study provides a first step in comparing neural processes related to $\mathrm{AVH}$ in psychotic and nonpsychotic individuals, these results cannot explain if the same pathophysiological mechanism gives rise to AVH in psychotic and nonpsychotic individuals with $\mathrm{AVH}$ as the resulting activation patterns may reflect a final common pathway triggered by different mechanisms. Therefore, these results cannot be used as support for or against the continuum hypothesis of psychosis in which it is usually assumed that AVH in all individuals result from the same pathophysiological mechanisms. To elucidate if AVH in different groups indeed arise from the same underlying pathology, future research should focus on comparing groups with and without a history of $\mathrm{AVH}$, while they are not actively hallucinating, for instance using resting state functional connectivity or structural anatomical measures. Together with the present results such research would shed more light on identical or different pathophysiology in both groups.

To our knowledge, this is the first study comparing brain activation during AVH between nonpsychotic and psychotic individuals with AVH. In addition, as far as the authors are concerned, no studies compared brain activation during AVH between different patients groups including patients with schizotypal and borderline personality disorder, bipolar disorder, and psychotic depression. As such a comparison could provide additional clues regarding similarities and differences in brain activation during AVH in different groups; future studies should focus on comparing AVH-related brain activation between patients with different diagnoses.

Activation of bilateral frontal, temporoparietal, and motor areas during $\mathrm{AVH}$ is largely in line with previous studies in psychotic patients. ${ }^{11-14}$ Thus far, only one study investigated brain activation during AVH in nonclinical individuals. ${ }^{15}$ This study included seven nonclinical individuals with AVH and 7 control subjects. The main difference between this study and the current study is that the current study compared AVH between psychotic and nonpsychotic individuals, while Linden and colleagues ${ }^{15}$ compared brain activation during $\mathrm{AVH}$ in nonclinical individuals with brain activation during imagery in a control group. As Linden and colleagues ${ }^{15}$ reported activation of frontotemporal language areas, in the left hemisphere and their contralateral homologues and the supplementary motor area during $\mathrm{AVH}$, as well as during verbal imagery, these results are for the most part in concordance with the present study.

While activation of motor areas, as observed in this study, most likely results from the employed balloonsqueeze paradigm, ${ }^{32}$ the role of bilateral frontal and temporoparietal regions in the experience of AVH is not yet clear. A number of theoretical frameworks, which have been proposed to account for the experience of $\mathrm{AVH}$ may aid in interpreting which cognitive functions are represented by activation of these areas during AVH.

First, the most influential, contemporary, model proposes that AVH occur due to a failure to recognize self-generated inner speech. ${ }^{33,34}$ Consequently, left hemisphere language production and perception areas, as well as regions implicated in self-monitoring, play a crucial role in this model. Activation of left frontal and temporoparietal regions as observed during $\mathrm{AVH}$ 
has consistently been implicated in language perception and production processes, ${ }^{35-38}$ providing support for this hypothesis. However, this study provides no information regarding the self-monitoring aspect of this theory. Support for this comes from a number of studies investigating self-recognition in patients with AVH. ${ }^{39,40}$ In addition, this theory cannot explain activation of right hemisphere frontal and temporoparietal regions, which is also observed during $\mathrm{AVH}$.

A second model proposes AVH to result from the release of language activity in the right hemisphere, which is normally inhibited in the healthy brain. ${ }^{41}$ While right hemisphere frontal and temporoparietal areas are not considered classical language regions, previous studies showed that the right hemisphere can produce so-called nonpropositional or "automatic" language, consisting of highly over learned sequences of low linguistic complexity. ${ }^{42,43}$ Activation of right hemisphere frontal and temporoparietal language regions is in line with this model; however, this model does not incorporate involvement of left hemisphere language regions, as observed in this study.

Furthermore, a third theory states that AVH result from aberrant activation of the primary auditory cortex. ${ }^{44}$ As this region was not significantly activated in the current study, this study provides no support to this theory.

Finally, the fourth model hypothesizes that AVH result from spontaneous memory recollection, leading to the reexperience of previously encoded information. $^{45,46}$ As no activation of regions implicated in memory processes was observed, this study does not support this model. However, previous studies reported involvement of the parahippocampal region, implicated in memory processes, prior to AVH. ${ }^{12,47}$ Perhaps, memory recollection preceding AVH triggers activations in language-related areas responsible for the experience of the actual AVH.

Another cognitive process likely to be involved in the experience of AVH consists of cue detection in which the AVH represent the detected cues. Previous studies showed that bilateral frontal and temporoparietal regions, observed in this study, have been implicated in the detection of salient events, providing support for the involvement of this function in $\mathrm{AVH} .{ }^{48,49}$

From this discussion, it is clear that a number of cognitive processes may be involved in AVH including cue detection, language production and perception processes, memory, and motor processes. Consequently, future studies should focus on disentangling which cognitive domains are involved in the experience of $\mathrm{AVH}$, favorably by directly comparing $\mathrm{AVH}$ to a number of cognitive tasks, tapping into the aforementioned cognitive domains. An intuitive expectation is that multiple cognitive processes are involved in the experience of AVH. For instance, spontaneous memory recollection may activate language-related areas in both hemispheres, leading to the reexperience of this memory, which is subsequently detected by regions involved in the detection of salient events. Finally, self-recognition deficits may explain why this memory is not recognized as such, rendering the belief that the content of the AVH comes from an outside source. Unfortunately, investigating brain activation during $\mathrm{AVH}$ does not allow one to separate brain activation associated with the experience of AVH from activation involved in the genesis of this phenomenon. In addition, one should be careful in comparing brain activation during AVH to activation elicited by a cognitive task such as a language paradigm, as both vary considerably. For instance, when studying $\mathrm{AVH}$, frequency, duration, and content of the "presented" stimuli differ between participants while during cognitive tasks these variables are generally held constant.

Surprisingly, we were not able to replicate earlier findings with respect to the significant correlation between lateralization of language regions during the experience of AVH and the emotional valence of the content of the voices. ${ }^{14}$ This may be one reason that, even though a significant difference in emotional valence was obtained, no significant difference between brain activation was obtained between the two groups.

\section{Limitations}

These results should be interpreted with some caution as possible differences between the groups may not have become visible, due to for instance the fact that fMRI BOLD does not demonstrate all types of neuronal activation, ${ }^{50}$ nor does it do so at finer spatial and temporal scales. Furthermore, the moderate number of subjects may have been of influence as it was previously reported that about 25 subjects are necessary to achieve $80 \%$ power in fMRI studies. ${ }^{51}$ However, as not even a trend could be observed when comparing the groups, sample size would most probably have to be increased dramatically to detect significant differences between the groups. To enable a thorough comparison between the two groups, we substantially increased sensitivity for detecting (even small) differences by using a hypothesis-based approach (small volume correction) in which only voxels in regions hypothesized to be involved in AVH were tested for differences between the groups. Sensitivity was increased as this analysis dramatically decreases the number of comparisons in comparison to testing for all the voxels in the brain. Finally, a limitation of this study is that both groups were highly selected and can therefore not readily be generalized to psychotic and nonpsychotic individuals in the general population.

In summary, during the experience of $\mathrm{AVH}$, psychotic and nonpsychotic individuals activated a common set of brain regions, implicating the involvement of the same cortical network in the experience of AVH in both groups. 


\section{Funding}

Nederlandse Wetenschappelijke Organisatie (Dutch Scientific Research Organization) (916.56.172)

\section{Acknowledgments}

All authors state that there are no conflicts of interest.

\section{References}

1. Sigdwick H, Johnson A, Myers FWH, Podmore F, Sigdwick E. Report on the census of hallucinations. In: Proceedings of the Society for Psychical Research. London. Vol XXVI. London: Kegan Paul, Trench, Trübner \& Co; 1894.

2. Sartorius N, Jablensky A, Korten A, et al. Early manifestations and first-contact incidence of schizophrenia in different cultures. A preliminary report on the initial evaluation phase of the WHO Collaborative Study on determinants of outcome of severe mental disorders. Psychol Med. 1986;16:909-928.

3. Tien AY. Distributions of hallucinations in the population. Soc Psychiatry Psychiatr Epidemiol. 1991;26:287-292.

4. Sommer IE, Daalman K, Rietkerk T, et al. Healthy individuals with auditory verbal hallucinations; who are they? Psychiatric assessments of a selected sample of 103 subjects. Schizophr Bull. 2008;36:633-641.

5. Johns LC, Nazroo JY, Bebbington P, Kuipers E. Occurrence of hallucinatory experiences in a community sample and ethnic variations. Br J Psychiatry. 2002;180:174-178.

6. van Os J, Hanssen M, Bijl RV, Ravelli A. Strauss (1969) revisited: a psychosis continuum in the general population? Schizophr Res. 2000;45:11-20.

7. van Os J, Linscott RJ, Myin-Germeys I, Delespaul P, Krabbendam L. A systematic review and meta-analysis of the psychosis continuum: evidence for a psychosis proneness-persistence-impairment model of psychotic disorder. Psychol Med. 2009;39:179-195.

8. Daalman K, Boks MP, Diederen KM, et al. Auditory verbal hallucinations in healthy and psychotic individuals. J Clin Psychiatry. In press.

9. Honig A, Romme MA, Ensink BJ, Escher SD, Pennings MH, deVries MW. Auditory hallucinations: a comparison between patients and nonpatients. J Nerv Ment Dis. 1998;186:646-651.

10. David AS. Why we need more debate on whether psychotic symptoms lie on a continuum with normality. Psychol Med. 2010;40:1935-1942.

11. Allen P, Laroi F, McGuire PK, Aleman A. The hallucinating brain: a review of structural and functional neuroimaging studies of hallucinations. Neurosci Biobehav Rev. 2008;32:175-191.

12. Diederen KM, Neggers SF, Daalman K, et al. Deactivation of the parahippocampal gyrus preceding auditory hallucinations in schizophrenia. Am J Psychiatry. 2010;167:427-435.

13. Jardri R, Pouchet A, Pins D, Thomas P. Cortical activations during auditory verbal hallucinations in schizophrenia: a coordinate-based meta-analysis. Am J Psychiatry. 2011;168:73-81.

14. Sommer IE, Diederen KM, Blom JD, et al. Auditory verbal hallucinations predominantly activate the right inferior frontal area. Brain. 2008;131(pt 12):3169-3177.

15. Linden DE, Thornton K, Kuswanto CN, Johnston SJ, van de Ven V, Jackson MC. The brain's voices: comparing nonclinical auditory hallucinations and imagery. Cereb Cortex. 2011; 21:330-337.

16. Diederen KM, De Weijer AD, Daalman K, et al. Decreased language lateralization is characteristic of psychosis, not auditory hallucinations. Brain. 2010;133(pt 12):3734-3744.

17. van Lutterveld R, Oranje B, Kemner C, et al. Increased psychophysiological parameters of attention in non-psychotic individuals with auditory verbal hallucinations. Schizophr Res. 2010;121:153-159.

18. Laroi F, Marczewski P, Van der Linden M. Further evidence of the multi-dimensionality of hallucinatory predisposition: factor structure of a modified version of the Launay-Slade Hallucinations Scale in a normal sample. Eur Psychiatry. 2004;19:15-20.

19. Slotema CW. Can low-frequency repetitive transcranial magnetic stimulation really relieve medication-resistant auditory verbal hallucinations? Negative results from a large randomized controlled trial. Biol Psychiatry. 2011;69:450-456.

20. Andreasen NC, Flaum M, Arndt S. The comprehensive assessment of symptoms and history (CASH). An instrument for assessing diagnosis and psychopathology. Arch Gen Psychiatry. 1992;49:615-623.

21. First MB, Spitzer RL, Gibbon M, Williams JBW. The structured clinical interview for DSM-III-R personality-disorders (SCID-II) .1. Description. J Pers Disord. 1995;9:83-91.

22. Raine A. The SPQ: a scale for the assessment of schizotypal personality based on DSM-III-R criteria. Schizophr Bull. 1991;17:555-564.

23. Endicott J, Spitzer RL, Fleiss JL, Cohen J. The global assessment scale. A procedure for measuring overall severity of psychiatric disturbance. Arch Gen Psychiatry. 1976;33:766-771.

24. Kay SR, Fiszbein A, Opler LA. The positive and negative syndrome scale (PANSS) for schizophrenia. Schizophr Bull. 1987;13:261-276.

25. Haddock G, McCarron J, Tarrier N, Faragher EB. Scales to measure dimensions of hallucinations and delusions: the psychotic symptom rating scales (PSYRATS). Psychol Med. 1999;29:879-889.

26. Neggers SF, Hermans EJ, Ramsey NF. Enhanced sensitivity with fast three-dimensional blood-oxygen-level-dependent functional MRI: comparison of SENSE-PRESTO and 2DEPI at 3 T. NMR Biomed. 2008;21:663-676.

27. Worsley KJ, Friston KJ. Analysis of fMRI time-series revisited-again. Neuroimage. 1995;2:173-181.

28. Nichols T, Brett M, Andersson J, Wager T, Poline JB. Valid conjunction inference with the minimum statistic. Neuroimage. 2005;25:653-660.

29. Tzourio-Mazoyer N, Landeau B, Papathanassiou D, et al. Automated anatomical labeling of activations in SPM using a macroscopic anatomical parcellation of the MNI MRI single-subject brain. Neuroimage. 2002;15:273-289.

30. Genovese CR, Lazar NA, Nichols T. Thresholding of statistical maps in functional neuroimaging using the false discovery rate. Neuroimage. 2002;15:870-878.

31. Jansen A, Menke R, Sommer J, et al. The assessment of hemispheric lateralization in functional MRI-robustness and reproducibility. Neuroimage. 2006;33:204-217.

32. Shergill SS, Brammer MJ, Williams SC, Murray RM, McGuire PK. Mapping auditory hallucinations in schizophrenia using functional magnetic resonance imaging. Arch Gen Psychiatry. 2000;57:1033-1038.

33. Frith C. Positron emission tomography studies of frontal lobe function: relevance to psychiatric disease. Ciba Found Symp. 1991;163:181-191 discussion: 191-187. 
34. Frith C, Done J. Positive symptoms of schizophrenia. $\mathrm{Br} J$ Psychiatry. 1989;154:569-570.

35. Price CJ. The anatomy of language: a review of $100 \mathrm{fMRI}$ studies published in 2009. Ann N Y Acad Sci. 2010;1191:62-88.

36. Ferstl EC, Neumann J, Bogler C, von Cramon DY. The extended language network: a meta-analysis of neuroimaging studies on text comprehension. Hum Brain Mapp. 2008;29:581-593.

37. Bookheimer S. Functional MRI of language: new approaches to understanding the cortical organization of semantic processing. Annu Rev Neurosci. 2002;25:151-188.

38. Cabeza R, Nyberg L. Imaging cognition II: an empirical review of 275 PET and fMRI studies. J Cogn Neurosci. 2000;12:1-47.

39. Allen P, Aleman A, McGuire PK. Inner speech models of auditory verbal hallucinations: evidence from behavioural and neuroimaging studies. Int Rev Psychiatry. 2007;19:407-415.

40. Waters F, Woodward T, Allen P, Aleman A, Sommer I. Selfrecognition deficits in schizophrenia patients with auditory hallucinations: a meta-analysis of the literature. Schizophr Bull. 2010; doi: 10.1093/schbul/sbq144.

41. Sommer IE, Diederen KM. Language production in the nondominant hemisphere as a potential source of auditory verbal hallucinations. Brain. 2009;132:e124.

42. Winhuisen L, Thiel A, Schumacher B, et al. Role of the contralateral inferior frontal gyrus in recovery of language function in poststroke aphasia: a combined repetitive transcranial magnetic stimulation and positron emission tomography study. Stroke. 2005;36:1759-1763.
43. Van Lancker D, Cummings JL. Expletives: neurolinguistic and neurobehavioral perspectives on swearing. Brain Res Brain Res Rev. 1999;31:83-104.

44. Lennox BR, Park SB, Jones PB, Morris PG. Spatial and temporal mapping of neural activity associated with auditory hallucinations. Lancet. 1999;353:644.

45. Copolov DL, Seal ML, Maruff P, et al. Cortical activation associated with the experience of auditory hallucinations and perception of human speech in schizophrenia: a PET correlation study. Psychiatry Res. 2003;122:139-152.

46. Waters FA, Badcock JC, Michie PT, Maybery MT. Auditory hallucinations in schizophrenia: intrusive thoughts and forgotten memories. Cognit Neuropsychiatry. 2006;11:65-83.

47. Hoffman RE, Anderson AW, Varanko M, Gore JC, Hampson $\mathrm{M}$. Time course of regional brain activation associated with onset of auditory/verbal hallucinations. Br J Psychiatry. 2008; 193:424-425.

48. Hampshire A, Chamberlain SR, Monti MM, Duncan J, Owen AM. The role of the right inferior frontal gyrus: inhibition and attentional control. Neuroimage. 2010;50:1313-1319.

49. Corbetta M, Shulman GL. Control of goal-directed and stimulus-driven attention in the brain. Nat Rev Neurosci. 2002;3:201-215.

50. Logothetis NK, Pauls J, Augath M, Trinath T, Oeltermann A. Neurophysiological investigation of the basis of the fMRI signal. Nature. 2001;412:150-157.

51. Desmond JE, Glover GH. Estimating sample size in functional MRI (fMRI) neuroimaging studies: statistical power analyses. J Neurosci Methods. 2002;118:115-128. 